\title{
Expression of Osteoprotegrin Is Enhanced in Lung Cancer Tissues and Promotes Aggressive Cellular Traits in H3122 Lung Cancer Cells
}

\author{
ZHEN YU ${ }^{1,2,3}$, ANDREW J. SANDERS ${ }^{3}$, SIONED OWEN ${ }^{3}$, SHAN CHENG ${ }^{1,2}$, \\ XIAOMEI YANG ${ }^{1,2}$ and WEN G. JIANG ${ }^{1,2,3}$ \\ ${ }^{1}$ Department of Biochemistry and Molecular Biology, School of Basic Medical Sciences, \\ ${ }^{2}$ Beijing Key Laboratory of Cancer \& Metastasis Research, Capital Medical University, Beijing, P.R. China; \\ ${ }^{3}$ Cardiff China Medical Research Collaborative, Cardiff University School of Medicine, Cardiff, U.K.
}

\begin{abstract}
Background: Osteoprotegrin (OPG), a secreted protein and a member of the tumor necrosis factor receptor superfamily has been well-characterized and is an important regulator of bone remodeling by blocking osteoclast maturation thus preventing osteolysis. In recent years, OPG has been reported to have an association with the malignant capacity of various cancer types and cancerassociated bone metastasis, although the mechanisms of this are not clearly understood. Materials and Methods: In this study, OPG expression was analyzed in human lung cancer tissue and normal tissue based on the dataset of The Cancer Genome Atlas and Oncomine. The in vitro effect of OPG on H3122 lung cancer cells was also assessed by characterizing cell function following knock-down and forced overexpression in this cell line. Results: The expression of OPG was significantly increased in lung cancer tissues compared to the normal control group and $O P G$ promoted the malignant phenotypes of $H 3122$ cells in in vitro models. Conclusion: OPG may be a potential driver of lung cancer cells and therefore might have potential in therapy and diagnostics.
\end{abstract}

Non-small cell lung cancer (NSCLC) is the most common malignancy worldwide and results in a large number of cancer-related deaths globally, representing a substantial burden for patients and their families. Currently, lung cancer

Correspondence to: Wen G Jiang, Cardiff China Medical Research Collaborative, Cardiff University School of Medicine, Heath Park, Cardiff, CF14 4XN, U.K. E-mail: jiangw@cf.ac.uk; or XiaoMei Yang, Beijing Key Laboratory of Cancer \& Metastasis Research, Capital Medical University, Beijing 100069, PR China. E-mail: XMYang126@126.com.

Key Words: OPG, lung cancer, H3122, cell function. accounts for nearly $12 \%$ of all cancer, causing approximately 1.1 million deaths each year (1) and increases patients' morbidity and the associated economic burden.

Cancer-related deaths are frequently the result of metastasis to other sites. Commonly, lung cancer results in metastatic cancer colonization of the brain, skeleton and adrenal glands (2). Patients with metastases to secondary sites, especially to bone, have a high degree of morbidity, decreased quality of life and mortality.

Osteoprotegrin (OPG) is a secreted protein and a member of the tumor necrosis factor (TNF) receptor superfamily. It is expressed as a circulating glycoprotein of 401 amino acids with seven structural domains $(3,4)$ and has been wellcharacterized as a regulator of bone remodeling by blocking osteoclast maturation and preventing the breakdown of bone (5). The $O P G$ gene is located on chromosome 8 at the $8 \mathrm{q} 24$ position, which seems to harbor a gene cluster involved in the regulation of bone development and remodeling. Circulating OPG can be found either as a free monomer of $60-\mathrm{kDa}$ or as a disulphide bond-linked homodimer form of $120-\mathrm{kDa}$, which is usually biologically more hypocalcaemically active than the monomeric form $(3,6,7)$.

OPG as a secreted protein can be detected in serum as well as having the capacity to act as decoy receptor for its ligands, receptor activator of nuclear factor kappa-B ligand (RANKL) and TNF-related apoptosis-inducing ligand (TRAIL). OPG binding to RANKL prevents receptor activator of nuclear factor kappa-B (RANK)-mediated osteoclast proliferation and maturation, thus maintaining bone remodeling in the bone forming phase. The OPG/RANKL ratio has been identified as an important determinant of skeletal bone mass and skeletal integrity (3). OPG has a weaker affinity for TRAIL but plays an important role in blocking the inducement of death receptors (DRs) 4 and 5 (8), thus providing a potential survival mechanism for a range of solid cancer cells (9-11). 
Recent research has shown that OPG may be a novel prognostic biomarker and a new therapeutic target for the treatment of patients with colorectal carcinoma and breast cancer $(12,13)$. In the current study, we examined the expression levels of OPG in lung cancer tissues and investigated the effect of targeting OPG on H3122 cell proliferation, adhesion, migration and invasion.

\section{Materials and Methods}

Bioinformatic analysis of OPG expression in cancer and normal tissues. We used the paired-sample $t$-test for multiple testing to identify the expression of OPG in lung adenocarcinoma tumor tissues $(n=517)$ and normal tissues $(n=59)$ from the Genome Atlas project (TCGA) database and in a range of lung cancers (adenocarcinoma $n=139$, carcinoid tumors $n=20$, small cell lung carcinoma $n=6$, squamous cell lung carcinoma $n=21$ ) from the Oncomine database.

Cell culture. Human NSCLC cell line, H3122, was obtained from the American Type Culture Collection (Manassas, VA, USA). The cells were cultured with RPMI medium 1640 basic supplemented with $10 \%$ fetal calf serum. (PAN BIOTECH, Aidenbach, Germany), streptomycin and penicillin (HyClone ${ }^{\mathrm{TM}}$, Logan, UT, USA) in a cell incubator at $37.0^{\circ} \mathrm{C}$, with $95 \%$ humidity and $5 \% \mathrm{CO}_{2}$.

Cell transfection. $\mathrm{H} 3122$ cells were transfected with OPG overexpression pENTER plasmid and OPG-hammerhead ribozyme pEF6/TOPO plasmid using the Polyplus DNA transfection reagent (Polyplus-transfection, Strasbourg, France). Transfected cells were harvested $48 \mathrm{~h}$ following OPG plasmid transfection and the western blot was used to verify the expression of OPG. Full sequence of $O P G$ was purchased from Vigene Biosciences (Rockville, MD, USA).

Western blot. Sodium dodecyl sulfate-polyacrylamide gel electrophoresis (SDS-PAGE)/western blotting was used to detect OPG protein expression in the H3122 lung cancer cells. Lysis buffer was first used to lyse confluent cells, then the protein was heated to $100^{\circ} \mathrm{C}$ in a water bath. Subsequently, the cell lysate proteins were separated using a $10 \%$ SDS-PAGE before blotting the proteins onto a nitrocellulose membrane, which was subsequently blocked with 5\% skimmed milk for 1 hour to block non-specific protein binding. The primary antibody to OPG (1:500) (AB9986, Abcam, Cambridge, UK) was used to probe OPG protein. Glyceraldehyde 3-phosphate dehydrogenase (GAPDH) was detected as a loading control at a concentration of 1:1,000 (ZSGB-BIO, Beijing, CHINA), The ECL system (Fusion $\mathrm{Fx}$, Vilber Lourmat, France) was used to visualize and analyze the protein bands.

In vitro cell growth assay. Cells were plated into a 96-well plate at a density of 2,000 cells $/ 200 \mu 1 /$ well. Cell growth was assessed after 5 days incubation (overnight, day 2, 3 and 5, respectively). A cell counting kit (CCK8, Dojindo, Kamimashiki-gun, Kumamoto, Japan) was used to evaluate cell viability. Cell density was assessed using a spectrophotometer (BioTek, Winooski, VT, USA) at a wavelength of $450 \mathrm{~nm}$.
Wounding/migration assay. Cells were plated at a density of $1 \times 10^{6}$ cells/well in 6-well plates and cultured overnight. Following confluence, a $10 \mu \mathrm{l}$ pipette tip was used to scratch a wound in the monolayer before washing with phosphate-buffered saline (PBS) twice to remove floating cells. The cells were subsequently photographed using an inverted microscope to observe cellular movement to heal the wound. Image-J software (National Institutes of Health, Bethesda, MD, USA) was used to analyze the migration of the cells at the 0 and 24 hour time points.

Cell matrix adhesion assay. A 96-well plate was pre-coated with Matrigel (BD Biosciences, Oxford, UK) at a concentration of $5 \mu \mathrm{g} / \mathrm{well}$, diluted in serum-free medium and dried in an oven at $37^{\circ} \mathrm{C}$. After rehydrating the Matrigel membrane in serum-free medium, $4 \times 10^{5}$ cells were seeded into each well and the plates incubated for $40 \mathrm{~min}$. Following incubation, non-adherent cells were removed through PBS washes and remaining adherent cells were fixed in $4 \%$ formalin and subsequently stained with $0.5 \%$ crystal violet $(\mathrm{w} / \mathrm{v})$. Representative images were captured prior to crystal violet solubilization in $10 \%$ acetic acid and absorbance was quantified at $540 \mathrm{~nm}$ on a plate reading spectrophotometer (BioTek).

In vitro invasion assay. Transwell inserts with $8 \mu \mathrm{m}$ pores (Corning Inc., Corning, NY, USA) were coated with $50 \mu \mathrm{g}$ Matrigel which was subsequently dried at $37^{\circ} \mathrm{C}$ in an oven. After rehydration in serum-free medium for $40 \mathrm{~min}$ at room temperature, $2 \times 10^{4}$ cells were seeded into the insert in medium containing $10 \%$ fetal bovine serum (FBS) and $1 \mathrm{ml}$ medium containing 10\% FBS medium was added to the bottom chamber before incubating the plates for 2 days. Following incubation, cells that had invaded through the matrix and adhered to the underside of the insert were fixed in $4 \%$ formalin and subsequently stained in $0.5 \%(\mathrm{w} / \mathrm{v})$ crystal violet. Following this, crystal violet stain was extracted and dissolved from the invaded cell using $10 \%$ acetic acid and measured using a spectrophotometer at 540nm (BioTek).

Statistical analysis. All experimental function assays were repeated at least three independent times. Representative data are presented with standard deviation. The results of the in vitro assays were assessed using the non-paired (two-sided) Student's $t$-test or oneway ANOVA test using SPSS 19.0 software (IBM Inc., Armonk, NY, USA). A $p$-value of less than 0.05 was defined as statistically significant.

\section{Results}

Bioinformatic analysis of OPG expression in online databases. The expression of OPG was analyzed from the Cancer Genome Atlas project (TCGA) lung cancer database and Oncomine database. Overall, we found that the expression of OPG was higher in all of the lung cancer tissues compared with normal tissues, suggesting that OPG may be a malignant factor in lung cancer (Figure 1).

Establishment of OPG overexpression and knock-down in H3122 cells. To study the effect of OPG in H3122 lung cancer cells, we overexpressed and knocked-down OPG in this cell line. Western blot analysis was used to verify 
A

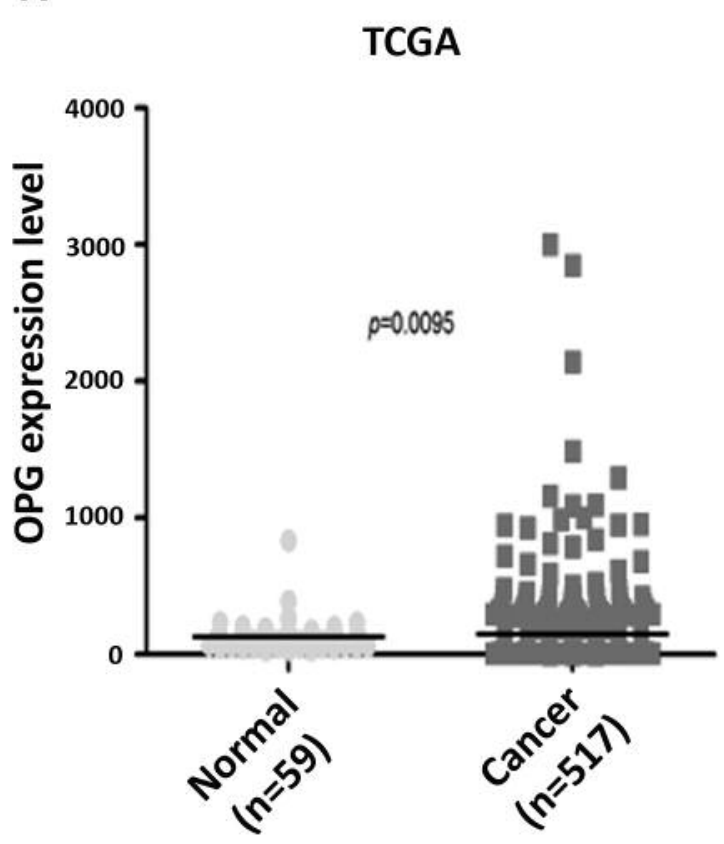

B

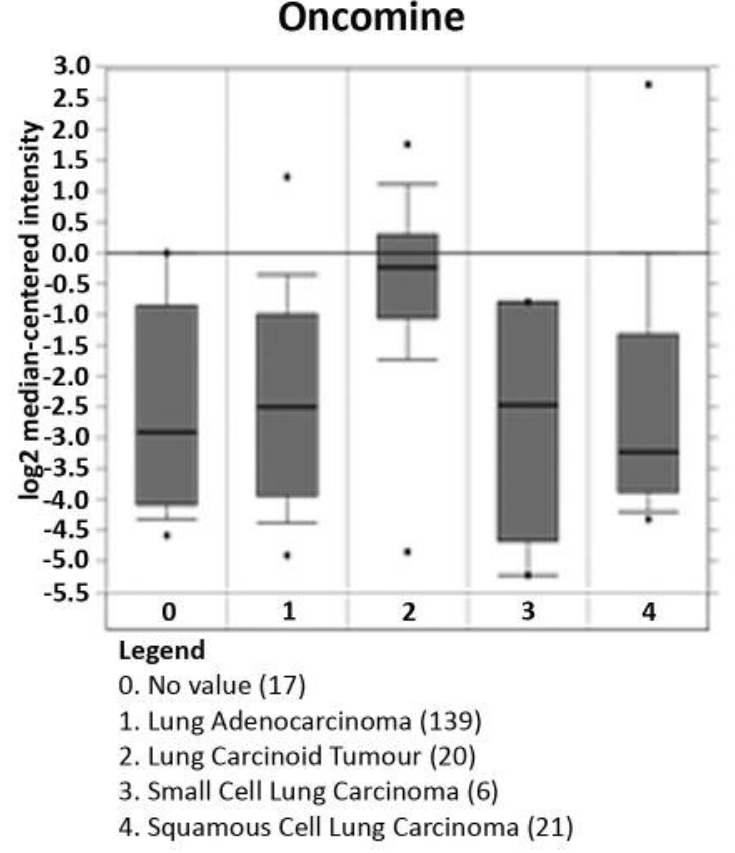

Figure 1. A: The expression of osteoprotegrin (OPG) was analyzed in the Cancer Genome Atlas project (TCGA) lung cancer database, where a significant elevation in OPG expression was observed in lung adenocarcinoma cancer tissue $(n=517)$ compared to normal tissues $(n=59, p<0.001)$. $B$ : The expression of OPG was also analyzed in the Oncomine database where lung adenocarcinoma ( $n=139)$, carcinoid tumor ( $n=20)$ and small cell lung carcinoma $(n=6)$ were all found to be enhanced in comparison to normal tissue $(n=17, p<0.05)$.

overexpression and suppression of OPG following transfection with appropriate plasmids. OPG protein was strongly expressed in the H3122-overexpressing cells, and markedly reduced in the H3122-knockdown cells in comparison to control cells (Figure 2A and B). This trend was further confirmed following semi-quantitative analysis of the protein band and comparison to control cells (Figure $2 \mathrm{C}$ and $\mathrm{D})$.

Functional characterization of OPG overexpression and knock-down in H3122 cells. Compared with the control groups, the overexpression of OPG promoted the malignant phenotype of human H3122 cells, significantly enhancing cellular proliferation at the fourth and the fifth days (Figure $3 \mathrm{~A}$, both $p<0.001 v s$. vector control), cell matrix- adhesion (Figure 3B, $p<0.05 v s$. vector control), cell migration (Figure $3 \mathrm{C}, p<0.05 v s$. vector control) and cell invasion (Figure 3D, $p<0.01 v s$. vector control). In keeping with this, the knockdown of OPG expression in H3122 cells lead to a significant reduction in five day growth (Figure 4A, $p<0.001 v s$. vector control), cell adhesion (Figure $4 \mathrm{~B} p<0.05 v s$. vector control), migration (Figure $4 \mathrm{C}, p<0.05 v s$. vector control) and invasion (Figure 4D, $p<0.001 v s$. vector control). These results strongly suggested that OPG is involved in the regulation of growth, adhesion, migration and invasion of lung cancer cells.

\section{Discussion}

In characterizing the role of OPG, studies have focused on the role of OPG in bone development and remodeling. OPG can block RANKL-induced osteoclastogenesis and some preclinical studies and clinical studies have investigated the therapeutic potential of recombinant OPG in cancer-related osteolysis (14).

As research shows, OPG is not only present in bone tissue but also in many others, such as the heart, arteries, veins, kidney, liver, spleen, thymus and lymph nodes (15). In this study, we found that the expression of OPG was higher in lung cancer tissues compared to normal tissues from the TCGA database. These datasets support similar trends that have been reported in a wide range of solid tumors, including prostate, breast, pancreatic and colorectal (16-18). Hence, these trends of increased OPG expression in malignant tissues indicates that OPG may be an indicator of malignancy, although its precise role in cancer formation requires further investigation. 
A

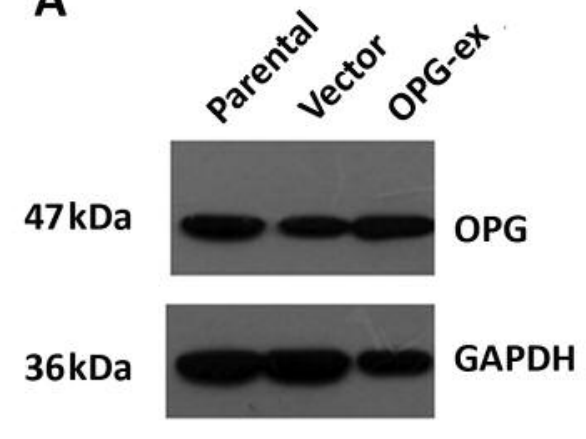

C

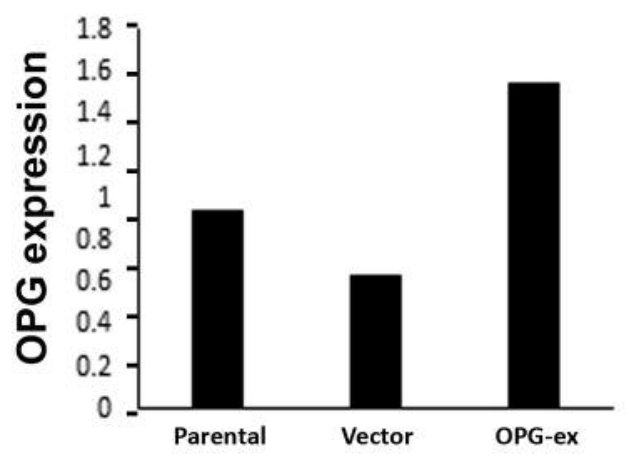

B

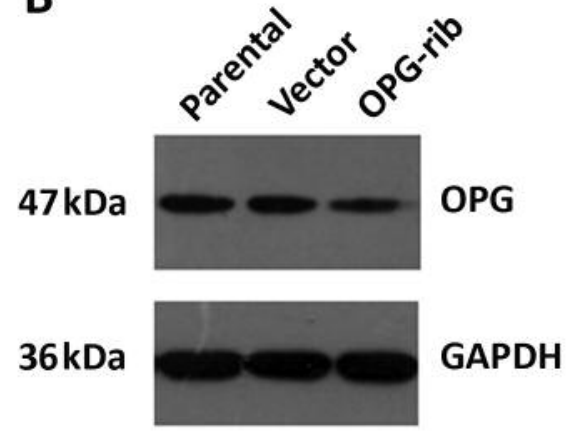

D

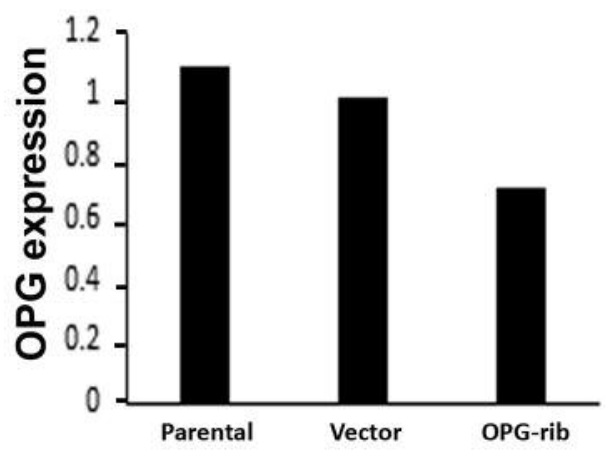

Figure 2. Western blot analysis demonstrating the protein expression of osteoprotegrin (OPG) following its overexpression (OPG-ex) (A) and knockdown (OPG-rib) (B). OPG expression was found to be enhanced in the H3122 OPG-ex group and decreased in the H3122 OPG-rib group in comparison to the control parental and vector control groups. C, D: Semi-quantitative analysis of band intensity and normalization against GAPDH was also used to confirm this trend.

OPG is also considered as a survival factor for tumor cells inhibiting tumor cell apoptosis (6). In vitro studies suggest that OPG exerts tumor-promoting effects by binding to TRAIL, thereby preventing induction of apoptosis (19). As it has been found to be expressed in neovessels associated with malignant tumors and in angiogenic microvessels associated with inflammatory osteolytic diseases, OPG has also been considered to be a factor affecting tumor angiogenesis (20). Research also shows that OPG-mediated angiogenic activities are mediated by mitogen-activated protein kinase, protein kinase B and mammalian target of the rapamycin signaling cascade (21).

Additionally, our results showed that the overexpression of OPG was linked to the elevation of growth, adhesion, invasion and migration of $\mathrm{H} 3122$ cells in vitro. Conversely, OPG knock-down in H3122 cells, can have an impact on cell functions, can reduce cell growth, adhesion, invasion and migration. Other in vitro studies in different tumor types have shown that OPG can influence key cancer traits and cellular response to external factors such as hepatocyte growth factor and stromal cell-derived factor $1(13,22,23)$.

Research has also shown that OPG is linked with poorer prognosis in other tumor types. In a study of 103 gastric adenocarcinoma tissues, high levels of $O P G$ gene expression correlated with increased invasion and metastasis, and predicted poor prognosis (24). This trend has also been reported in prostate cancer (25-27).

Further evidence also suggests that an elevated level of OPG detected in serum from patient with lung cancer, amongst others, may in the future have diagnostic or prognostic potential $(28,29)$.

In conclusion, we have demonstrated that increased OPG was detected in human lung cancer tissue compared to normal tissue. Furthermore we demonstrated that further increasing OPG expression in H3122 NSCLC in vitro promotes key cancer traits, including migration and invasion. These observations suggest that OPG may have potential as a biomarker and clinical target in lung cancer, warranting further study into the biological role of OPG in lung cancer. 
A

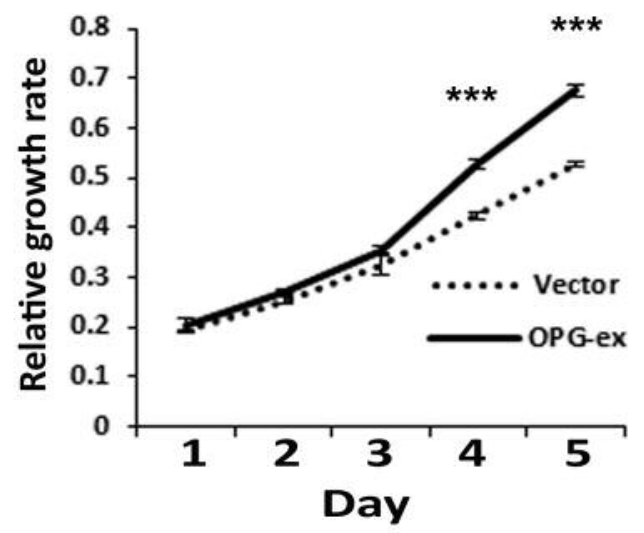

C $\mathbf{O h}$

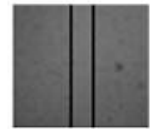

$24 \mathrm{~h}$

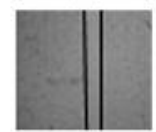

\section{ing}

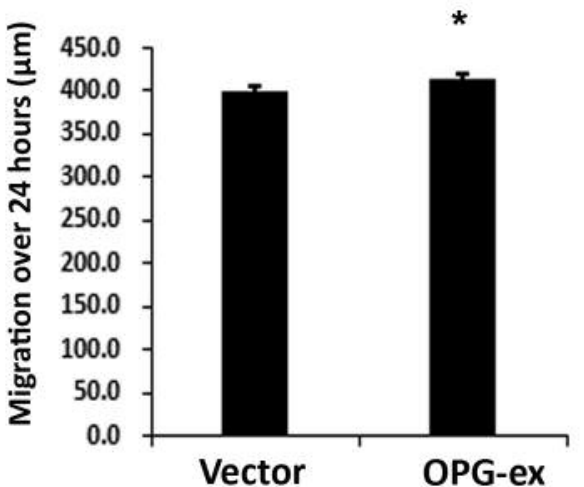

B
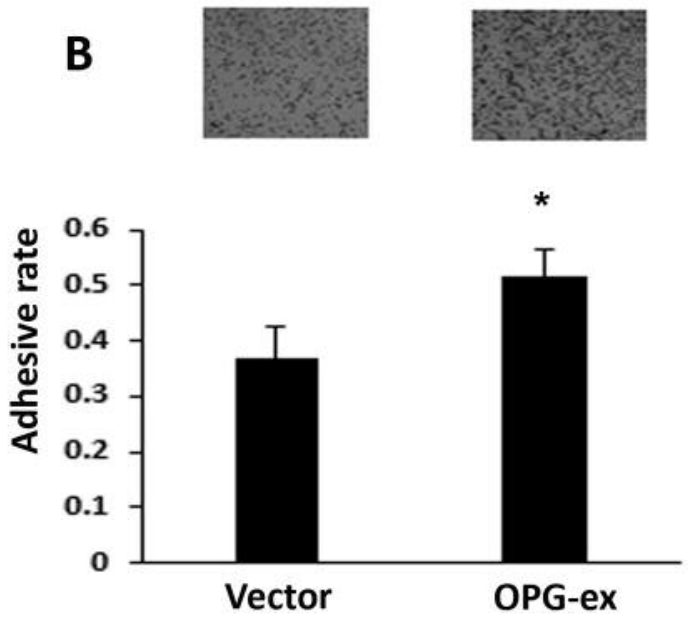

D
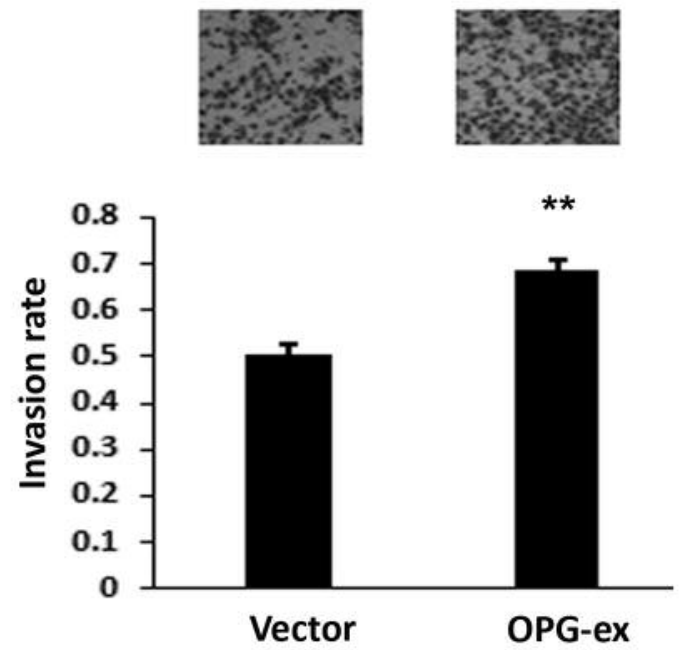

Figure 3. Characterization of the role of osteoprotegrin $(O P G)$ overexpression in $H 3122$ cell growth $(A)$, matrix adhesion $(B)$, migration $(C)$ and invasion (D). The overexpression of OPG (OPG-ex) promoted $H 3122$ cell growth over both the fourth and the fifth days compared to the control group $(* * * p<0.001)$. OPG overexpression also promoted cell matrix adhesion when compared to control group cells $(* p<0.05)$ and increased H3122 cellular migration in comparison to vector control cells $(* p<0.05)$. A similar enhancement of invasive capacity was also seen in comparison to vector control cells following OPG overexpression $\left({ }^{* *} p<0.01\right)$. Representative images are shown of at least three independent replicates.

\section{Acknowledgements}

The Authors wish to express their heartfelt gratitude to Capital Medical University; Cancer Research Wales and Cardiff University China Medical Scholarship for supporting this work.

\section{References}

1 Reck M, Heigener DF, Mok T, Soria JC and Rabe KF: Management of non-small-cell lung cancer: recent developments. Lancet 382: 709-719, 2013.
2 Popper HH: Progression and metastasis of lung cancer. Cancer Metastasis Rev 35: 75-91, 2016.

3 Khosla S: Minireview: the OPG/RANKL/RANK system. Endocrinology 142: 5050-5055, 2001.

4 Maginn EN, Browne PV, Hayden P, Vandenberghe E, MacDonagh B, Evans P, Goodyer M, Tewari P, Campiani G, Butini S, Williams DC, Zisterer DM, Lawler MP and McElligott AM: PBOX-15, a novel microtubule targeting agent, induces apoptosis, upregulates death receptors, and potentiates TRAILmediated apoptosis in multiple myeloma cells. Br J Cancer 104: 281-289, 2011. 

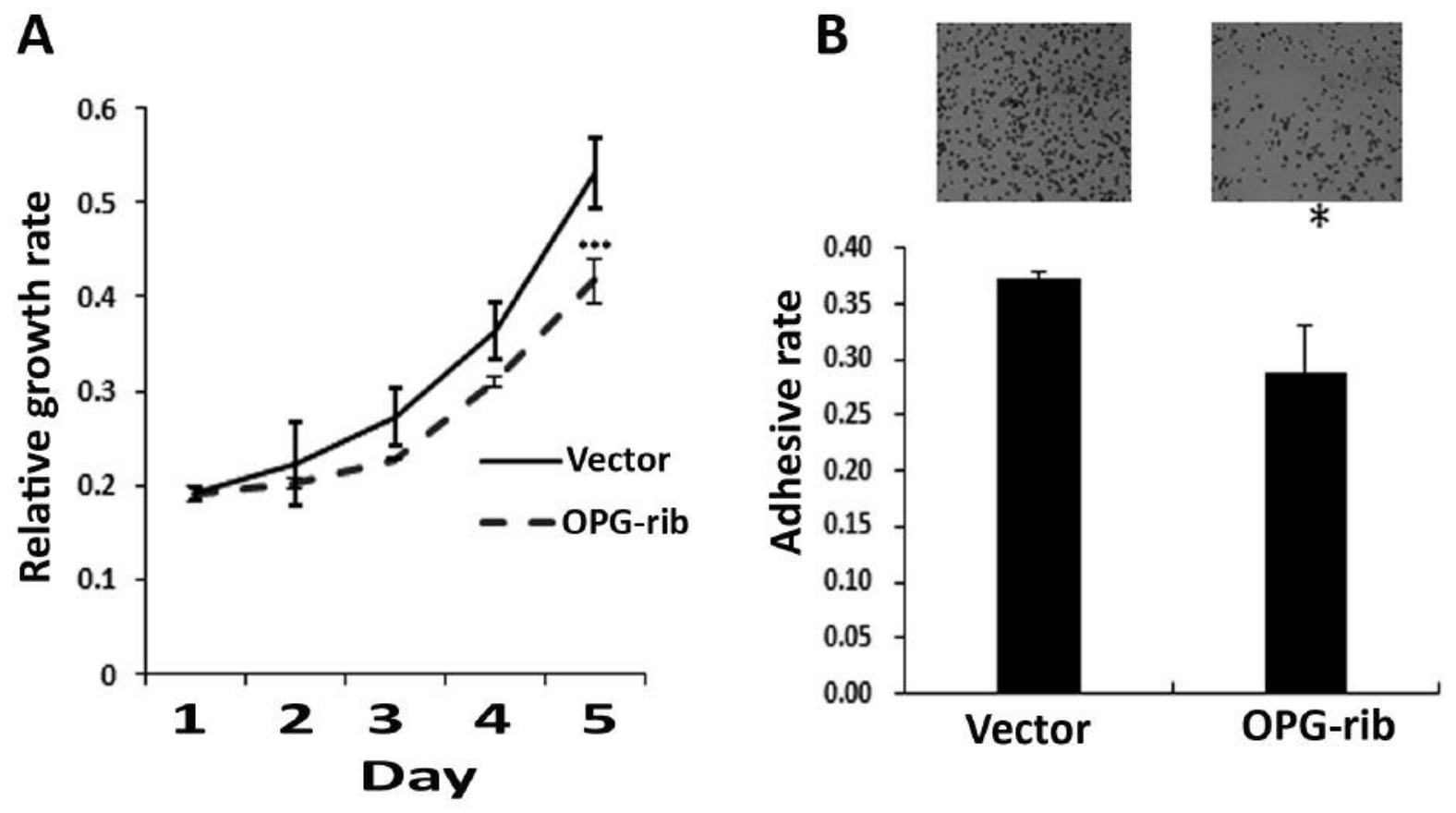

C
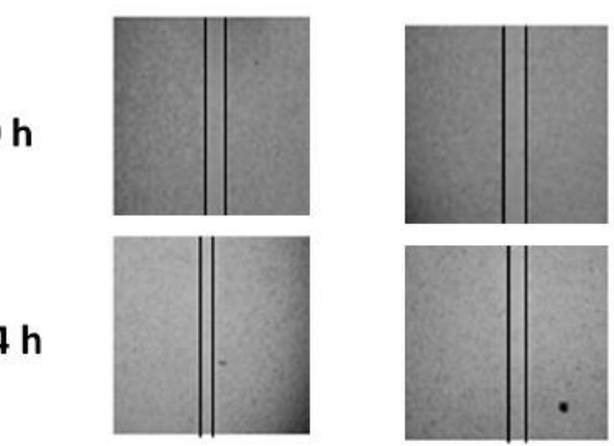

$\mathrm{Oh}$


Figure 4. Characterization of the role of osteoprotegrin (OPG) knock-down (OPG-rib) in H3122 cell growth (A), matrix-adhesion (B), migration $(C)$ and invasion $(D)$. The knock-down of OPG inhibited $H 3122$ cell growth at the fifth day of incubation compared to the control group $(* * * p<0.001)$. OPG knockdown also inhibited cell matrix adhesion compared to the vector control $(* p<0.05)$ and reduced H3122 cellular migration over 24 hours in comparison to vector control cells $(* p<0.05)$. A similar inhibition of invasive capacity was also seen in comparison to vector control cells following OPG knock-down $\left({ }^{* *} p<0.001\right)$. Representative images are shown of at least three independent replicates. 
5 Weichhaus M, Chung ST and Connelly L: Osteoprotegerin in breast cancer: beyond bone remodeling. Mol Cancer 14: 117, 2015.

6 Holen I and Shipman CM: Role of osteoprotegerin (OPG) in cancer. Clinical Science 110: 279-291, 2006.

7 Schoppet M, Preissner KT, and Hofbauer LC: RANK ligand and osteoprotegerin: paracrine regulators of bone metabolism and vascular function. Arteriorscler Thromb Vasc Biol 22: 549-553, 2002.

8 Wang S and El-Deiry WS: TRAIL and apoptosis induction by TNF-family death receptors. Oncogene 22: 8628-8633, 2003.

9 De Toni EN, Thieme SE, Herbst A, Behrens A, Stieber P, Jung A, Blum H, Goke B and Kolligs FT: OPG is regulated by beta-catenin and mediates resistance to TRAIL-induced apoptosis in colon cancer. Clin Cancer Res 14: 4713-4718, 2008.

10 Holen I, Croucher PI, Hamdy FC and Eaton CL: Osteoprotegerin (OPG) is a survival factor for human prostate cancer cells. Cancer Res 62: 1619-1623, 2002.

11 Neville-Webbe HL, Cross NA, Eaton CL, Nyambo R, Evans CA, Coleman RE and Holen I: Osteoprotegerin (OPG) produced by bone marrow stromal cells protects breast cancer cells from TRAILinduced apoptosis. Breast Cancer Res Treat 86: 269-279, 2004.

12 Baud'huin M, Duplomb L, Teletchea S, Lamoureux F, RuizVelasco C, Maillasson M, Redini F, Heymann MF, and Heymann D: Osteoprotegerin: multiple partners for multiple functions. Cytokine Growth Factor Rev 24: 401-409, 2013.

13 Bucay N, Sarosi I, Dunstan CR, Morony S, Tarpley J, Capparelli C, Scully S, Tan HL, Xu W, Lacey DL, Boyle WJ, and Simonet WS: osteoprotegerin-deficient mice develop early onset osteoporosis and arterial calcification. Genes Dev 12: 12601268, 1998.

14 Lane D, Matte I, Rancourt C and Piche A: Osteoprotegerin (OPG) protects ovarian cancer cells from TRAIL-induced apoptosis but does not contribute to malignant ascites-mediated attenuation of TRAIL-induced apoptosis. J Ovarian Res 5: 34, 2012.

15 Higgs JT, Jarboe JS, Lee JH, Chanda D, Lee CM, Deivanayagam $\mathrm{C}$ and Ponnazhagan S: Variants of osteoprotegerin lacking TRAIL binding for therapeutic bone remodeling in osteolytic malignancies. Mol Cancer Res 13: 819-827, 2015.

16 Brown JM, Corey E, Lee ZD, True LD, Yun TJ, Tondravi M and Vessella RL: Osteoprotegerin and rank ligand expression in prostate cancer. Urology 57: 611-616, 2001.

17 Reid P and Holen I: Pathophysiological roles of osteoprotegerin (OPG). Eur J Cell Biol 88: 1-17, 2009.

18 Shi W, Qiu W, Wang W, Zhou X, Zhong X, Tian G and Deng A: Osteoprotegerin is up-regulated in pancreatic cancers and correlates with cancer-associated new-onset diabetes. Biosci Trends 8: 322-326, 2014.
19 Malyankar UM, Scatena M, Suchland KL, Yun TJ, Clark EA and Giachelli CM: Osteoprotegerin is an alpha vbeta 3-induced, NFkappa B-dependent survival factor for endothelial cells. J Biol Chem 275: 20959-20962, 2000.

20 Kobayashi-Sakamoto M, Isogai E, Hirose K and Chiba I: Role of alphav integrin in osteoprotegerin-induced endothelial cell migration and proliferation. Microvas Res 76: 139-144, 2008.

21 Kobayashi-Sakamoto M, Isogai E and Holen I: Osteoprotegerin induces cytoskeletal reorganization and activates FAK, Src, and ERK signaling in endothelial cells. Eur J Haematol 85: 26-35, 2010.

22 Benslimane-Ahmim Z, Pereira J, Lokajczyk A, Dizier B, GalyFauroux I, Fischer AM, Heymann D and Boisson-Vidal C: Osteoprotegerin regulates cancer cell migration through SDF$1 / \mathrm{CXCR} 4$ axis and promotes tumor development by increasing neovascularization. Cancer Lett 395: 11-19, 2017.

23 Owen S, Sanders AJ, Mason MD and Jiang WG: Importance of osteoprotegrin and receptor activator of nuclear factor kappaB in breast cancer response to hepatocyte growth factor and the bone microenvironment in vitro. Int J Oncol 48: 919-928, 2016.

24 Truneh A, Sharma S, Silverman C, Khandekar S, Reddy MP, Deen KC, McLaughlin MM, Srinivasula SM, Livi GP, Marshall LA, Alnemri ES, Williams WV, and Doyle ML: Temperature-sensitive differential affinity of TRAIL for its receptors. DR5 is the highest affinity receptor. J Biol Chem 275(30): 23319-23325, 2000.

25 Chen G, Sircar K, Aprikian A, Potti A, Goltzman D and Rabbani SA: Expression of RANKL/RANK/OPG in primary and metastatic human prostate cancer as markers of disease stage and functional regulation. Cancer 107: 289-298, 2006.

26 Eaton CL, Wells JM, Holen I, Croucher PI and Hamdy FC: Serum osteoprotegerin (OPG) levels are associated with disease progression and response to androgen ablation in patients with prostate cancer. Prostate 59: 304-310, 2004.

27 Jung K, Lein M, Stephan C, Von Hosslin K, Semjonow A, Sinha P, Loening SA and Schnorr D: Comparison of 10 serum bone turnover markers in prostate carcinoma patients with bone metastatic spread: diagnostic and prognostic implications. Int $\mathbf{J}$ Cancer 111: 783-791, 2004.

$28 \mathrm{Lu} \mathrm{C}$, Sun C and Jin H: Serum sRANKL and sRANKL/OPG ratio: Novel biomarkers in non-small cell lung cancer. Oncol Lett 11: 2261-2265, 2016.

29 Zang L, Ma M, Hu J, Qiu H, Huang B and Chu T: The effects of lung and prostate cancer bone metastasis on serum osteoprotegerin levels: a meta-analysis. Sci Rep 5: 18324, 2015.

Received April 6, 2017

Revised April 20, 2017

Accepted April 25, 2017 